\title{
Release of Per- and polyfluoroalkyl Substances (PFASs) from Carpet and Clothing in Model Anaerobic Landfill Reactors
}

\section{Supporting Information}

Johnsie R. Lang ${ }^{a^{*}}$, B. McKay Allred ${ }^{\mathrm{b}}$, Graham F. Peaslee ${ }^{\mathrm{c}}$, Jennifer A. Field ${ }^{\mathrm{d}}$, Morton A.

Barlaz $^{\mathrm{a}}$

*Corresponding Author

${ }^{a}$ Department of Civil, Constructional, and Environmental Engineering

North Carolina State University

Box 7908

Raleigh, NC 27695-7908

J. R. Lang email: jrlang@ @ ncsu.edu

M. A. Barlaz email: barlaz@ncsu.edu

Phone: 919-515-7212

FAX: 919-515-7908

${ }^{\mathrm{b}}$ Department of Chemistry

Oregon State University

153 Gilbert Hall

Corvallis, Oregon 97331-4003

Email: allredb@onid.orst.edu

${ }^{\mathrm{c}}$ Department of Chemistry

Hope College

35 E. $12^{\text {th }}$ St.

Holland, MI 49423-3605

Email: peaslee@hope.edu

${ }^{\mathrm{d}}$ Department of Environmental and Molecular Toxicology

Oregon State University

1007 ALS Bldg.

2750 Campus Way

Corvallis, OR, 97331-4003

Email: jennifer.field@oregonstate.edu 


\section{Contents}

Table S1: Masses and volumes of materials added to carpet, clothing, and copy paper only reactors ........ 4

Table S2: Standards used during with LC/MS/MS analysis

Table S3: Initial concentration ranges of PFAS classes in anaerobic reactors (Day 0). See Table 1 for specific analytes included in each PFAS compound class. ${ }^{a, b}$

Figure S1: Methane production in carpet (A) live 1 and (B) live 2 during operation demonstrating four distinct phases: (I) lag phase, (II) exponential growth, (III) stationary, and (IV) stabilization phase......... 2

Figure S2: Methane production in clothing (A) live 1 and (B) live 2 during operation demonstrating four distinct phases: (I) lag phase, (II-I) first exponential growth, (III) stationary, and (II-II) second exponential growth phase. In contrast to the carpet reactors (Figure S1), these reactors were not monitored through the stabilization phase.

Figure S3: Reactor $\mathrm{pH}$ during anaerobic decomposition for $(\mathbf{A})$ carpet, $(\mathbf{B})$ clothing, and $(\mathbf{C})$ copy paper reactors

Figure S4. PFAS leaching from carpet live 1 presented as total concentrations at each sampling point. Methane $\left(\mathrm{CH}_{4}\right)$ measurement from the previous sampling point was presented if a measurement was not made on the same day as PFAS samples. Note the non-linear $\mathrm{x}$-axis.

Figure S5. PFAS leaching from clothing live 1 presented as the total concentration at each sampling point. Note non-linear $\mathrm{X}$-axis.

Figure S6. PFAS leaching from clothing abiotic 2 presented as the total concentration at each sampling point. Note non-linear $\mathrm{x}$-axis. 
PIGE Analysis of Reactor Solids. Individual clothing samples were analyzed with particle induced gamma-ray emission (PIGE) spectroscopy using a modified version of previously published methods to determine total $\mathrm{F}$ in biological and aerosol samples. ${ }^{1-4}$ In brief, the presence of fluorine in a material was determined by the reaction of an accelerated beam of protons striking its surface. The excitation that occurs in ${ }^{19} \mathrm{~F}$ nuclei from interactions with the accelerated ion beam causes the emission of a series of characteristic gamma rays, which can be used to determine the quantity of ${ }^{19} \mathrm{~F}$ nuclei in the target material. For this analysis, a beam of $3.4 \mathrm{MeV}$ protons from the Hope College Ion Beam Analysis Laboratory was extracted into air through a thin foil, and used to measure the fluorine content of solid materials placed in the beam. Typically $\sim 2 \mu \mathrm{C}$ of beam was delivered over 180 seconds to each target, and two characteristic ${ }^{19} \mathrm{~F}$ gamma rays (of $110 \mathrm{keV}$ and $197 \mathrm{keV}$ ) were detected at an angle of $110^{\circ}$ with respect to the beam by a high-purity Germanium detector ( $20 \%$ efficiency). These peaks were integrated after background subtraction and used to provide a quantitative measure (in arbitrary units of counts/ $\mu \mathrm{C}$ ) of fluorine content in each sample. The absolute detection limit for fluorine is on the order of ppm for this technique on fabric samples, but absolute calibration with respect to external standards is still on-going. Reproducibility across the triplicate samples analyzed was better than $10 \%$.

1. Lavielle, S., Gionnet, K., Ortega, R., Devès, G., Kilarski, V., Wehbe, K., Bikfalvi, A., \& Déléris, G. First Quantitative Imaging of Organic Fluorine within Angiogenic Tissues by Particle Induced Gamma-Ray Emission (PIGE) Analysis: First PIGE Organic Fluorine Imaging. Pharmaceutics. 2011. 3, 88-106.

2. Carvalho, M. L., Karydas, A. G., Casaca, C.; Zarkadas, C., Paradellis, T., Kokkoris, M.; Nsouli, B., \& Cunha, A. S. Fluorine determination in human healthy and carious teeth using the PIGE technique. Nucl. Instru. Meth. 2001, B179, 561-567.

3. Calastrini, F., Del Carmine P., Lucarelli F., Mando, P. A., Prati, P., \& Zucchiatti, A. Externalbeam PIGE for fluorine determination in atmospheric aerosol. Nucl. Instr. Meth. 1998 B136-138, 975-980.

4. Samudralwar, D. L. \& Robertson, J. D. Determination of major and trace elements in bones by simultaneous PIXE PIGE analysis. J. Radioanalyt. Nucl. Chem. 1993 169, 259-267 
Table S1: Masses and volumes of materials added to carpet, clothing, and copy paper only reactors

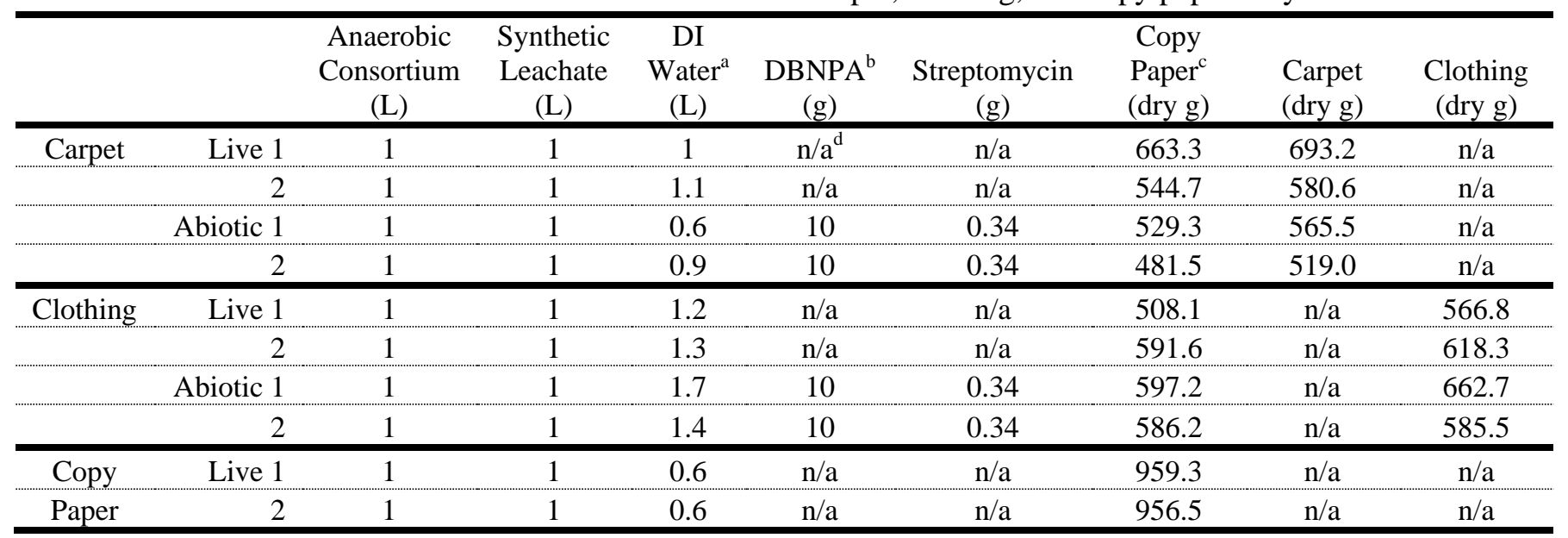
a. Deionized Water
b. 2,2-dibromo-3-nitrilopropionamide
c. Volume of moisture in the sample was not presented as it was less than $0.1 \mathrm{~L}$ in all reactors
d. $\mathrm{n} / \mathrm{a}=$ Not added 
Table S2: Standards used during with LC/MS/MS analysis

\begin{tabular}{|c|c|c|c|}
\hline Analyte & Standard Supplier (purity) & Internal Standard & Data Quality Level \\
\hline PFBS & Wellington $(>98 \%)$ & {$\left[{ }^{18} \mathrm{O}_{2}\right] \mathrm{PFHxS}$} & $\mathrm{Qn}^{\mathrm{a}}$ \\
\hline PFPS & Wellington (residual) & {$\left[{ }^{18} \mathrm{O}_{2}\right] \mathrm{PFHxS}$} & $\mathrm{Ql}^{\mathrm{b}}$ \\
\hline PFHxS & Wellington $(>98 \%)$ & {$\left[{ }^{18} \mathrm{O}_{2}\right] \mathrm{PFHxS}$} & Qn \\
\hline PFHpS & Wellington (residual) & {$\left[{ }^{13} \mathrm{C}_{2}\right] \mathrm{PFOS}$} & Q1 \\
\hline PFOS & Wellington (>98\%) & {$\left[{ }^{13} \mathrm{C}_{2}\right] \mathrm{PFOS}$} & Qn \\
\hline PFNS & Wellington (residual) & {$\left[{ }^{13} \mathrm{C}_{2}\right]$ PFOS } & Q1 \\
\hline PFDS & Wellington $(>98 \%)$ & {$\left[{ }^{13} \mathrm{C}_{2}\right]$ PFOS } & Qn \\
\hline 4:2 FTSA & Wellington (>98\%) & {$\left[{ }^{13} \mathrm{C}_{2}\right]$ 6:2 FTSA } & $\mathrm{Sq}^{\mathrm{c}}$ \\
\hline $6: 2$ FTSA & Wellington $(>98 \%)$ & {$\left[{ }^{13} \mathrm{C}_{2}\right]$ 6:2 FTSA } & Qn \\
\hline 8:2 FTSA & Wellington $(>98 \%)$ & {$\left[{ }^{13} \mathrm{C}_{2}\right]$ 6:2 FTSA } & $\mathrm{Sq}$ \\
\hline PFBA & Wellington $(>98 \%)$ & {$\left[{ }^{13} \mathrm{C}_{4}\right]$ PFBA } & Qn \\
\hline PFPeA & Wellington $(>98 \%)$ & {$\left[{ }^{13} \mathrm{C}_{3}\right] \mathrm{PFPeA}$} & Qn \\
\hline PFHxA & Wellington $(>98 \%)$ & {$\left[{ }^{13} \mathrm{C}_{2}\right] \mathrm{PFHxA}$} & Qn \\
\hline PFHpA & Wellington $(>98 \%)$ & {$\left[{ }^{13} \mathrm{C}_{4}\right] \mathrm{PFOA}$} & Qn \\
\hline PFOA & Wellington $(>98 \%)$ & {$\left[{ }^{13} \mathrm{C}_{4}\right] \mathrm{PFOA}$} & Qn \\
\hline PFNA & Wellington $(>98 \%)$ & {$\left[{ }^{13} \mathrm{C}_{5}\right]$ PFNA } & Qn \\
\hline PFDA & Wellington $(>98 \%)$ & {$\left[{ }^{13} \mathrm{C}_{2}\right] \mathrm{PFDA}$} & Qn \\
\hline PFUnDA & Wellington (>98\%) & {$\left[{ }^{13} \mathrm{C}_{2}\right] \mathrm{PFUnDA}$} & Qn \\
\hline PFDoDA & Wellington (>98\%) & {$\left[{ }^{13} \mathrm{C}_{2}\right]$ PFDoDA } & Qn \\
\hline PFTriDA & Wellington $(>98 \%)$ & {$\left[{ }^{13} \mathrm{C}_{2}\right]$ PFDoDA } & $\mathrm{Sq}$ \\
\hline PFTeDA & Wellington $(>98 \%)$ & {$\left[{ }^{13} \mathrm{C}_{2}\right]$ PFDoDA } & Q1 \\
\hline PFPDA & Wellington (residual) & {$\left[{ }^{13} \mathrm{C}_{2}\right]$ PFDoDA } & $\mathrm{Sq}$ \\
\hline PFHxDA & Wellington $(>98 \%)$ & {$\left[{ }^{13} \mathrm{C}_{2}\right]$ PFDoDA } & Q1 \\
\hline PFHpDA & Wellington (residual) & {$\left[{ }^{13} \mathrm{C}_{2}\right]$ PFDoDA } & $\mathrm{Sq}$ \\
\hline PFOcDA & Wellington $(>98 \%)$ & $\left.{ }^{13} \mathrm{C}_{2}\right]$ PFDoDA & Q1 \\
\hline 4:2 FTCA & $\mathrm{n} / \mathrm{a}^{\mathrm{d}}$ & {$\left[{ }^{13} \mathrm{C}_{2}\right]$ FHEA } & $\mathrm{Sc}^{\mathrm{e}}$ \\
\hline $6: 2$ FTCA & Wellington $(>98 \%)$ & {$\left[{ }^{13} \mathrm{C}_{2}\right]$ FHEA } & Qn \\
\hline $8: 2$ FTCA & Wellington $(>98 \%)$ & {$\left[{ }^{13} \mathrm{C}_{2}\right]$ FOEA } & Qn \\
\hline 10:2 FTCA & Wellington $(>98 \%)$ & {$\left[{ }^{13} \mathrm{C}_{2}\right]$ FDEA } & Qn \\
\hline 3:3 FTCA & Wellington $(>98 \%)$ & {$\left[{ }^{13} \mathrm{C}_{2}\right]$ FHEA } & Q1 \\
\hline 5:3 FTCA & Wellington (>98\%) & {$\left[{ }^{13} \mathrm{C}_{2}\right]$ FHEA } & Qn \\
\hline $7: 3$ FTCA & Wellington (>98\%) & {$\left[{ }^{13} \mathrm{C}_{2}\right]$ FOEA } & Qn \\
\hline 9:3 FTCA & $\mathrm{n} / \mathrm{a}$ & {$\left[{ }^{13} \mathrm{C}_{2}\right]$ FDEA } & $\mathrm{Sc}$ \\
\hline 4:2 FTUCA & $\mathrm{n} / \mathrm{a}$ & {$\left[{ }^{13} \mathrm{C}_{2}\right]$ FHUEA } & Sc \\
\hline 6:2 FTUCA & Wellington $(>98 \%)$ & {$\left[{ }^{13} \mathrm{C}_{2}\right]$ FHUEA } & Qn \\
\hline 8:2 FTUCA & Wellington $(>98 \%)$ & {$\left[{ }^{13} \mathrm{C}_{2}\right]$ FOEA } & Qn \\
\hline 10:2 FTUCA & $\mathrm{n} / \mathrm{a}$ & {$\left[{ }^{13} \mathrm{C}_{2}\right] \mathrm{FDEA}$} & $\mathrm{Sc}$ \\
\hline FBSAA & $\mathrm{n} / \mathrm{a}$ & {$\left[{ }^{13} \mathrm{C}_{2}\right] \mathrm{PFHxA}$} & $\mathrm{Sc}$ \\
\hline FPeSAA & $\mathrm{n} / \mathrm{a}$ & {$\left[{ }^{13} \mathrm{C}_{2}\right]$ PFHxA } & $\mathrm{Sc}$ \\
\hline FHxSAA & $\mathrm{n} / \mathrm{a}$ & {$\left[{ }^{2} \mathrm{H}_{3}\right] \mathrm{MeFOSAA}$} & $\mathrm{Sc}$ \\
\hline FHpSAA & $\mathrm{n} / \mathrm{a}$ & {$\left[{ }^{2} \mathrm{H}_{3}\right] \mathrm{MeFOSAA}$} & Sc \\
\hline FOSAA & Wellington (>98\%) & {$\left[{ }^{2} \mathrm{H}_{3}\right] \mathrm{MeFOSAA}$} & Qn \\
\hline MeFBSAA & $3 \mathrm{M}(\mathrm{n} / \mathrm{a})$ & {$\left[{ }^{13} \mathrm{C}_{2}\right] \mathrm{PFHxA}$} & $\mathrm{Sq}$ \\
\hline MeFPeSAA & $\mathrm{n} / \mathrm{a}$ & {$\left[{ }^{13} \mathrm{C}_{2}\right]$ PFHxA } & Sc \\
\hline MeFHxSAA & $\mathrm{n} / \mathrm{a}$ & {$\left[{ }^{2} \mathrm{H}_{3}\right] \mathrm{MeFOSAA}$} & $\mathrm{Sc}$ \\
\hline MeFHpSAA & $\mathrm{n} / \mathrm{a}$ & {$\left[{ }^{2} \mathrm{H}_{3}\right] \mathrm{MeFOSAA}$} & Sc \\
\hline MeFOSAA & Wellington $(>98 \%)$ & {$\left[{ }^{2} \mathrm{H}_{3}\right] \mathrm{MeFOSAA}$} & Qn \\
\hline EtFBSAA & $\mathrm{n} / \mathrm{a}$ & {$\left[{ }^{13} \mathrm{C}_{2}\right] \mathrm{PFHxA}$} & $\mathrm{Sc}$ \\
\hline EtFPeSAA & $\mathrm{n} / \mathrm{a}$ & {$\left[{ }^{13} \mathrm{C}_{2}\right]$ PFHxA } & Sc \\
\hline EtFHxSAA & $\mathrm{n} / \mathrm{a}$ & {$\left[{ }^{2} \mathrm{H}_{3}\right]$ EtFOSAA } & $\mathrm{Sc}$ \\
\hline
\end{tabular}




\begin{tabular}{llll} 
EtFHpSAA & n/a & {$\left[{ }^{2} \mathrm{H}_{3}\right]$ EtFOSAA } & Sc \\
EtFOSAA & Wellington $(>98 \%)$ & {$\left[{ }^{2} \mathrm{H}_{3}\right]$ EtFOSAA } & Qn \\
4/4 PFPIA & n/a & {$\left[{ }^{13} \mathrm{C}_{4}\right] 6: 2$ DiPAP } & Sc \\
4/6 PFPIA & Wellington (residual) & {$\left[{ }^{13} \mathrm{C}_{4}\right] 6: 2$ DiPAP } & Q1 \\
6/6 PFPIA & Wellington $(>98 \%)$ & {$\left[{ }^{13} \mathrm{C}_{4}\right] 6: 2$ DiPAP } & Qn \\
6/8 PFPIA & Wellington $(>98 \%)$ & {$\left[{ }^{13} \mathrm{C}_{4}\right] 6: 2$ DiPAP } & Qn \\
8/8 PFPIA & Wellington $(>98 \%)$ & {$\left[{ }^{13} \mathrm{C}_{4}\right] 6: 2$ DiPAP } & Qn \\
4/4 diPAP & n/a & {$\left[{ }^{13} \mathrm{C}_{4}\right] 6: 2$ DiPAP } & Sc \\
4/6 diPAP & n/a & {$\left[{ }^{13} \mathrm{C}_{4}\right] 6: 2$ DiPAP } & Sc \\
6/6 diPAP & Wellington $(>98 \%)$ & {$\left[{ }^{13} \mathrm{C}_{4}\right] 6: 2$ DiPAP } & Qn \\
6/8 diPAP & Wellington (residual) & {$\left[{ }^{13} \mathrm{C}_{4}\right] 8: 2$ DiPAP } & Q1 \\
8/8 diPAP & Wellington $(>98 \%)$ & {$\left[{ }^{13} \mathrm{C}_{4}\right] 8: 2$ DiPAP } & Qn \\
8/10 diPAP & Wellington (residual) & {$\left[{ }^{13} \mathrm{C}_{4}\right] 8: 2$ DiPAP } & Q1 \\
10/10 diPAP & Wellington (residual) & {$\left[{ }^{13} \mathrm{C}_{4}\right] 8: 2$ DiPAP } & Q1 \\
6/6 FTMAP & US FDA (n/a) & {$\left[{ }^{13} \mathrm{C}_{4}\right] 8: 2$ DiPAP } & Q1 \\
6/8 FTMAP & US FDA (n/a) & {$\left[{ }^{13} \mathrm{C}_{4}\right] 8: 2$ DiPAP } & Q1 \\
8/8 FTMAP & US FDA (n/a) & {$\left[{ }^{13} \mathrm{C}_{4}\right] 8: 2$ DiPAP } & Q1 \\
8/10 FTMAP & US FDA (n/a) & {$\left[{ }^{13} \mathrm{C}_{4}\right] 8: 2$ DiPAP } & Q1 \\
10/10 FTMAP & US FDA (n/a) & {$\left[{ }^{13} \mathrm{C}_{4}\right] 8: 2$ DiPAP } & Q1 \\
8/8 SAmPAP & US FDA (n/a) & {$\left[{ }^{13} \mathrm{C}_{4}\right] 8: 2$ DiPAP } & Q1 \\
\hline
\end{tabular}

a. Quantitative (Qn) PFASs has analytical standards and the measured accuracy fell within $90-110 \%$ and precision was $\leq 20 \%$ RSD.

b. For qualitative (Q1) PFASs, only a commercial reference material was available.

c. Semi-quantitative (Sq) PFASs has analytical standards, but the measured accuracy did not fall within 90$110 \%$ and/or the precision was $\geq 20 \% \mathrm{RSD}$.

d. Not applicable (n/a) as no standard was available.

e. For screen (Sc) PFASs, no reference material was available. 
Table S3: Initial concentration ranges of PFAS classes in anaerobic reactors (Day 0). See Table 1 for specific analytes included in each PFAS compound class. ${ }^{\mathrm{a} b}$

\begin{tabular}{|c|c|c|c|c|c|c|c|c|c|c|c|}
\hline \multicolumn{3}{|c|}{ nmol/L } & $\begin{array}{c}\sum \text { PFCAs } \\
(n=15)^{c}\end{array}$ & $\begin{array}{c}\sum \text { FTCAs } \\
(n=12)\end{array}$ & $\begin{array}{c}\sum \text { FTSAs } \\
(\mathbf{n}=3)\end{array}$ & $\begin{array}{c}\sum \text { DiPAPs } \\
(\mathbf{n}=7)\end{array}$ & $\begin{array}{c}\sum \text { PFSAs } \\
(n=7)\end{array}$ & $\begin{array}{c}\sum \text { FASAAs } \\
(n=15)\end{array}$ & $\begin{array}{c}\text { SAmPAP } \\
(n=1)\end{array}$ & $\begin{array}{c}\sum \text { PFPIAs } \\
(\mathbf{n}=\mathbf{5})\end{array}$ & $\begin{array}{c}\sum \text { PFASs } \\
(n=705\end{array}$ \\
\hline \multicolumn{2}{|c|}{ Copy Paper Only } & 1 & $<\mathrm{LOQ}^{\mathrm{d}}$ & ND & $<\mathrm{LOQ}$ & ND & $<\mathrm{LOQ}$ & ND & ND & ND & $<\mathrm{LOQ}$ \\
\hline Day 0 & Live & 2 & $\mathrm{ND}^{\mathrm{e}}$ & ND & $<\mathrm{LOQ}$ & ND & ND & ND & ND & ND & $<$ LO\& \\
\hline \multirow{4}{*}{$\begin{array}{l}\text { Carpet } \\
\text { Day } 0\end{array}$} & Live & 1 & 0.51 & 0.09 & $<\mathrm{LOQ}$ & 0.03 & 0.01 & 0.01 & $<\mathrm{LOQ}$ & $<$ LOQ & $0.66^{9}$ \\
\hline & & 2 & 0.82 & 0.07 & 0.01 & 0.05 & $<\mathrm{LOQ}$ & 0.02 & $<\mathrm{LOQ}$ & $<$ LOQ & 0.971 \\
\hline & Abiotic & 1 & 0.21 & $<\mathrm{LOQ}$ & ND & 0.01 & 0.01 & ND & ND & ND & 0.232 \\
\hline & & 2 & 0.51 & 0.01 & $<\mathrm{LOQ}$ & $<\mathrm{LOQ}$ & 0.11 & 0.01 & ND & ND & 0.633 \\
\hline \multirow{4}{*}{$\begin{array}{l}\text { Clothing } \\
\text { Day } 0\end{array}$} & Live & 1 & 0.13 & $<\mathrm{LOQ}$ & 0.02 & 0.02 & 0.02 & 0.01 & $<\mathrm{LOQ}$ & ND & 0.194 \\
\hline & & 2 & 2.28 & 0.56 & 1.95 & 0.01 & 0.03 & $<\mathrm{LOQ}$ & ND & ND & $4.84_{6}^{5}$ \\
\hline & Abiotic & 1 & 0.50 & 0.11 & 0.18 & 0.00 & 0.04 & ND & ND & ND & 0.837 \\
\hline & & 2 & 0.62 & 0.09 & 0.13 & $<\mathrm{LOQ}$ & 0.01 & ND & ND & ND & 0.848 \\
\hline
\end{tabular}

a. $\quad$ EFTMAPs $(n=5)$ were not presented because the measured concentration in all reactors was non-detect.

c. Single samples were analyzed for each time point, but the accuracy of the method and whole-method precision were previously determined to be $81-120 \%$ and 5.5-33\%, respectivelyThe number ( $n$ ) of analytes included in each PFAS compound class

d. LLOQ: All signals for analytes in compound class were less than the limit of quantification

e. ND: All signals for analytes in compound class were non-detect 
27 Table S4: Contribution of samples with and without analytical and/or internal standards at the final 28 sampling point

\begin{tabular}{|c|c|c|c|c|c|c|}
\hline & & & $\underline{\mathbf{Q n}^{\mathbf{a}}}$ & $\underline{S q}^{b}$ & $\mathrm{Ql}^{\mathbf{c}}$ & $\underline{\mathbf{S c}^{\mathrm{d}}}$ \\
\hline \multirow[t]{4}{*}{ Carpet } & \multirow[t]{2}{*}{ Live } & 1 & $98 \%$ & $2 \%$ & $<1 \%$ & $<1 \%$ \\
\hline & & 2 & $97 \%$ & $2 \%$ & $<1 \%$ & $<1 \%$ \\
\hline & \multirow{2}{*}{ Abiotic } & 1 & $98 \%$ & $2 \%$ & $<1 \%$ & $<1 \%$ \\
\hline & & 2 & $95 \%$ & $5 \%$ & $<1 \%$ & $<1 \%$ \\
\hline \multirow[t]{4}{*}{ Clothing } & \multirow[t]{2}{*}{ Live } & 1 & $89 \%$ & $2 \%$ & $9 \%$ & $<1 \%$ \\
\hline & & 2 & $87 \%$ & $13 \%$ & $<1 \%$ & $<1 \%$ \\
\hline & \multirow[t]{2}{*}{ Abiotic } & 1 & $73 \%$ & $27 \%$ & $<1 \%$ & $<1 \%$ \\
\hline & & 2 & $97 \%$ & $3 \%$ & $<1 \%$ & $<1 \%$ \\
\hline
\end{tabular}

a. Quantitative (Qn) PFASs has analytical standards and the measured accuracy fell within $90-110 \%$ and precision was $\leq 20 \%$ RSD.

b. Semi-quantitative (Sq) PFASs has analytical standards, but the measured accuracy did not fall within 90$110 \%$ and/or the precision was $\geq 20 \%$ RSD.

32

33

34

c. For qualitative (Ql) PFASs, only a commercial reference material was available.

d. For screen $(\mathrm{Sc})$ PFASs, no reference material was available. 
35 Figure S1: Methane production in carpet (A) live 1 and (B) live 2 during operation demonstrating four 36 distinct phases: (I) lag phase, (II) exponential growth, (III) stationary, and (IV) stabilization phase.

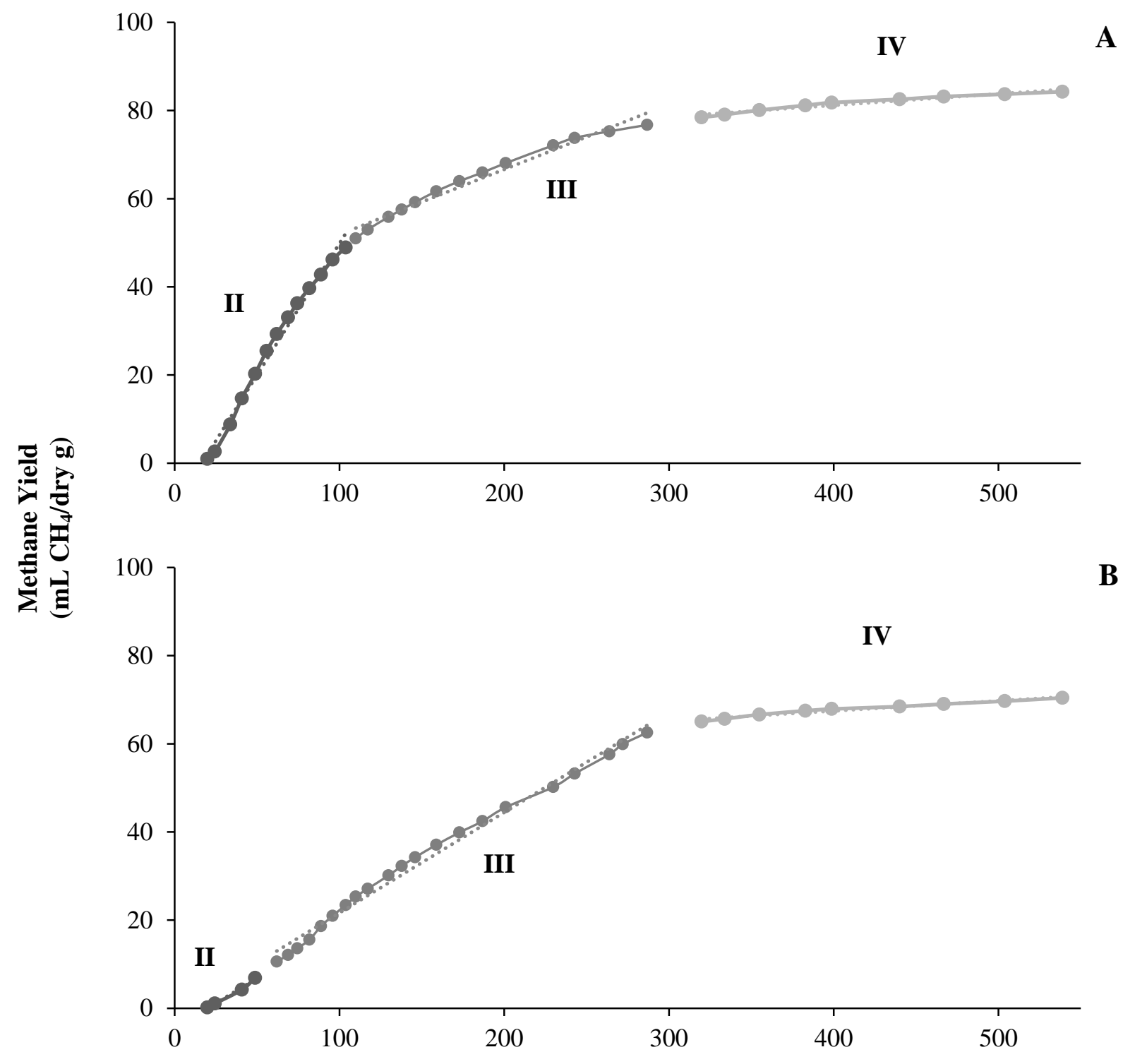

Day 
41 Figure S2: Methane production in clothing (A) live 1 and (B) live 2 during operation demonstrating four

42 distinct phases: (I) lag phase, (II-I) first exponential growth, (III) stationary, and (II-II) second

43 exponential growth phase. In contrast to the carpet reactors (Figure S1), these reactors were not monitored 44 through the stabilization phase.

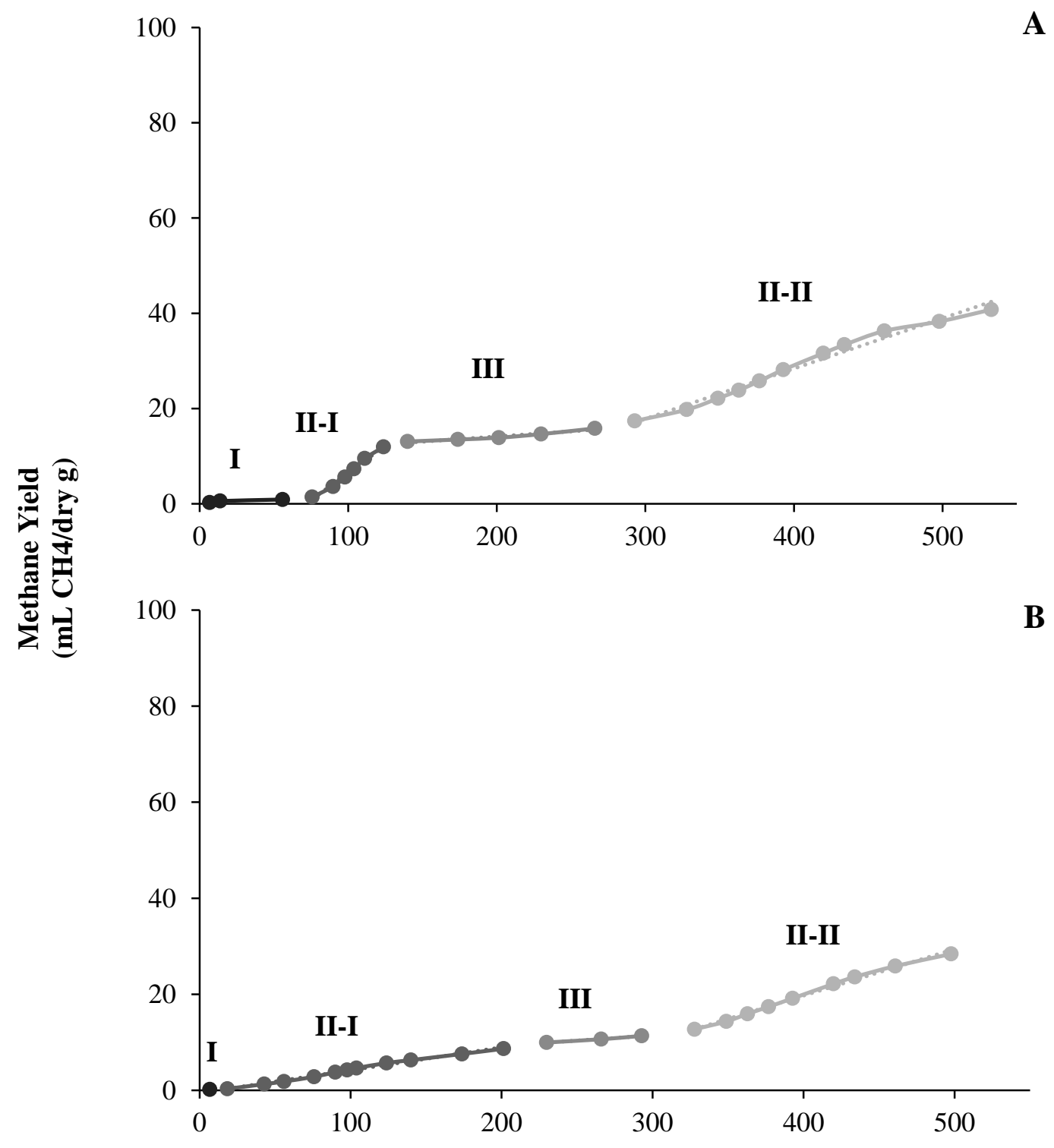

Day

45

46

47 
Figure S3: Reactor $\mathrm{pH}$ during anaerobic decomposition for (A) carpet, (B) clothing, and (C) copy paper reactors
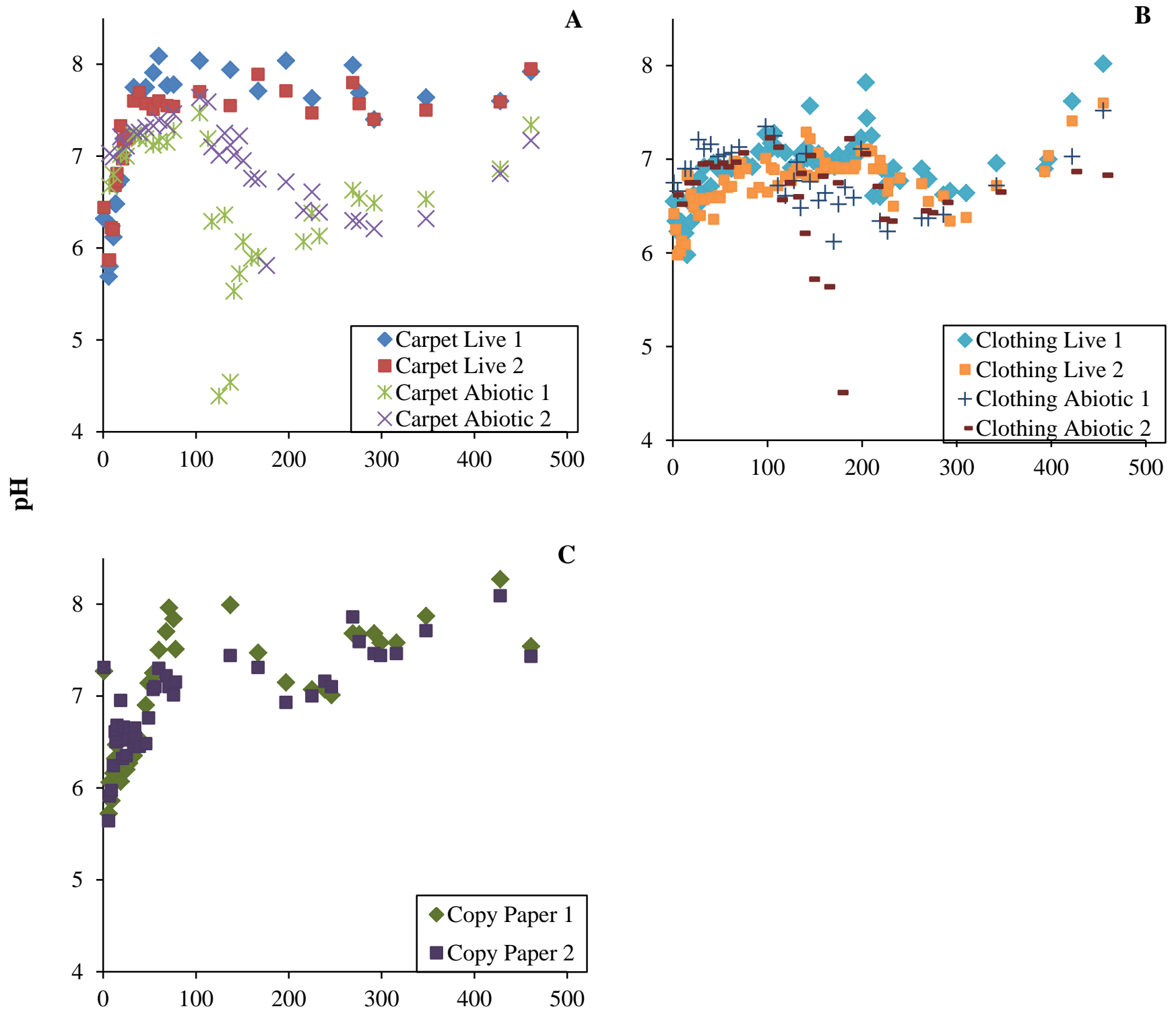

Day

50

51 
52 Figure S4. PFAS leaching from carpet live 1 presented as total concentrations at each sampling point.

53 Methane $\left(\mathrm{CH}_{4}\right)$ measurement from the previous sampling point was presented if a measurement was not

54 made on the same day as PFAS samples. Note the non-linear $\mathrm{x}$-axis.

55

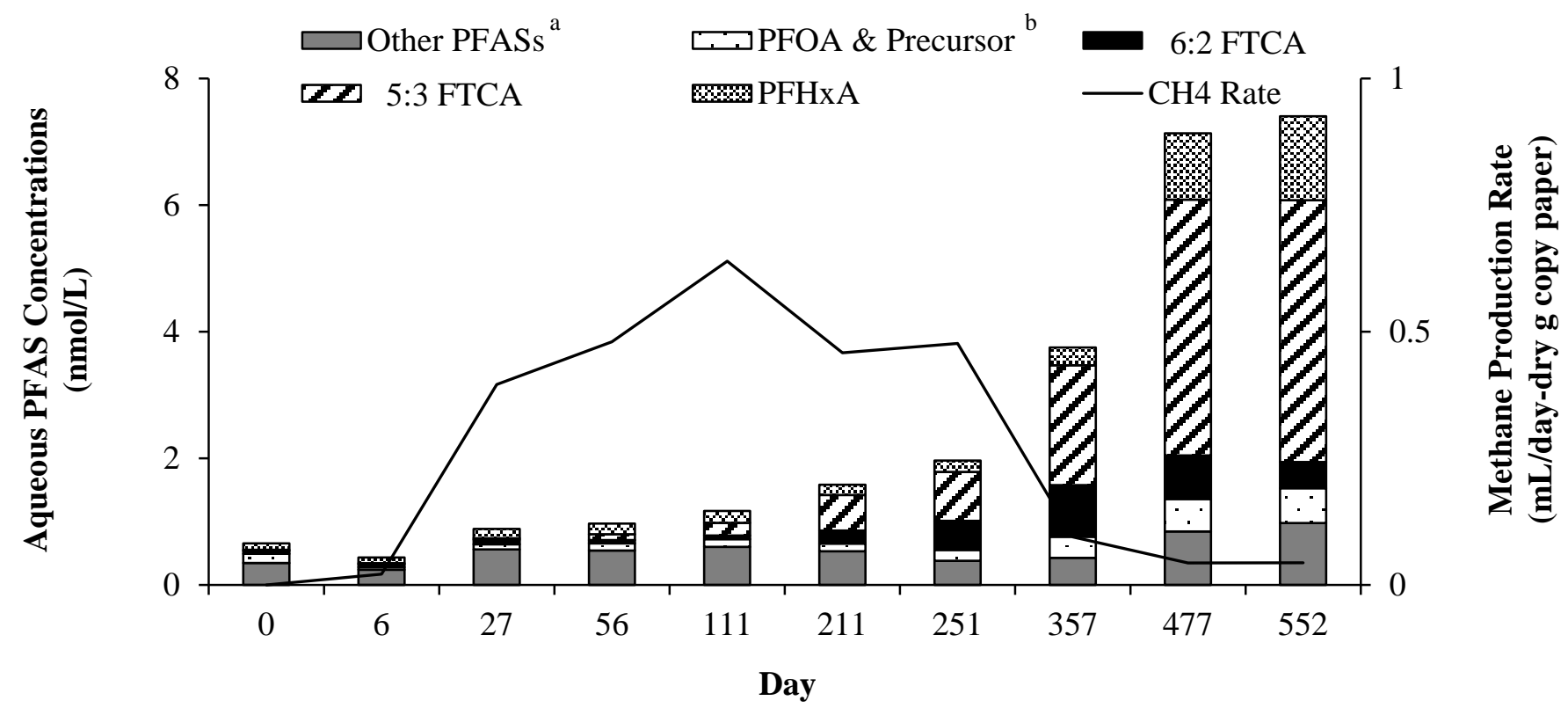

a. Other PFASs include the sum of concentrations for all other measured compounds $(\mathrm{n}=59)$

57

b. PFOA \& Precursors includes the sum of concentrations for PFOA, 8:2 FTCA, 8:2 FTUCA, 7:3 FTCA, 8:2 diPAPs, and 8:2 FTSA 
60 Figure S5. PFAS leaching from clothing live 1 presented as the total concentration at each sampling 61 point. Note non-linear x-axis.

62

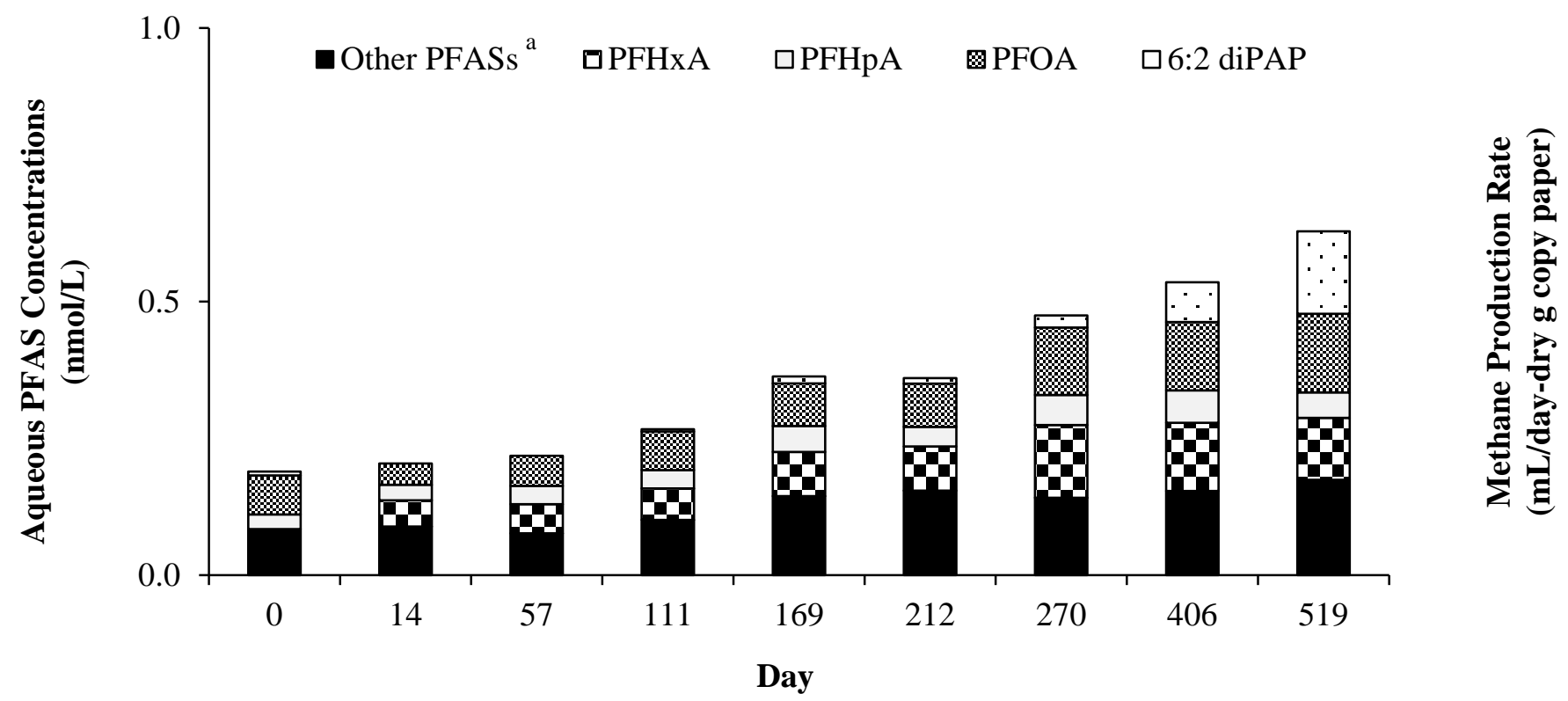

a. Other PFASs includes the sum concentrations for all other measured compounds $(n=66)$. 
65 Figure S6. PFAS leaching from clothing abiotic 2 presented as the total concentration at each sampling 66 point. Note non-linear x-axis.

67

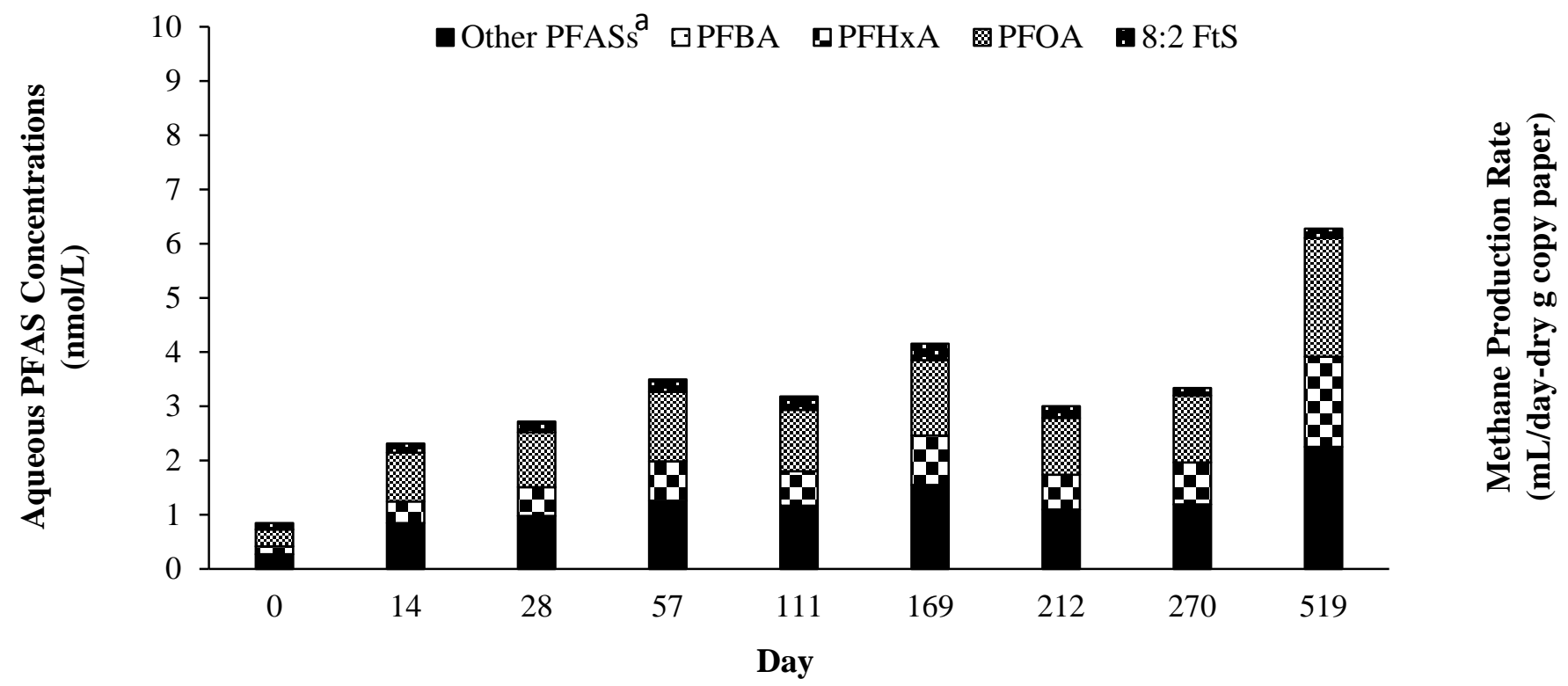

a. Other PFASs includes the sum concentrations for all other measured compounds $(n=66)$.

69 